\title{
Fluoroquinolone resistance and mutational profile of gyrA gene in pulmonary MDR tuberculosis patients
}

\section{Saba Kabir}

University of the Punjab, Lahore, Pakistan

\section{Zarfishan Tahir}

Institute of Public Health, Lahore, Pakistan

\section{Nadia Mukhtar}

University of Veterinary and Animal Sciences, Lahore

\section{Muhammad Sohail}

University of the Punjab, Lahore, Pakistan

Muhammad Saqalein

Government College University Faisalabad

Abdul Rehman ( $\square$ rehman.mmg@pu.edu.pk)

University of the Punjab

\section{Research article}

Keywords: XDR-Tuberculosis, Mycobacterium tuberculosis, flouroquinolone resistance, MTBDRsI assay

Posted Date: January 15th, 2020

DOI: https://doi.org/10.21203/rs.2.15811/v2

License: (1) This work is licensed under a Creative Commons Attribution 4.0 International License. Read Full License

Version of Record: A version of this preprint was published at BMC Pulmonary Medicine on May 11th, 2020. See the published version at https://doi.org/10.1186/s12890-020-1172-4. 


\section{Abstract}

Background Flouroquinolones (FQs) are the potential drugs that inhibit DNA synthesis and used in the treatment of MDR-TB and anti-TB short term regimens. In recent year's high proportion of flouroquinolone (FQs) resistance in Mycobacterium tuberculosis isolates has been observed. The development of FQs resistance among multidrug resistant TB (Pre-XDR TB) negatively impact patient treatment outcome and is a serious threat to control TB.

Methods A total of 562 samples were included in the study from patients with pulmonary TB which had been on anti-tuberculosis therapy. MTBDRsl assay was performed for molecular detection of mutations. Sequence analysis was performed for characterization and mutational profiling of FQ resistant isolates.

Results FQs resistance was observed in 104 (18.5\%) samples and most of them were previously treated and treatment failure cases. A total of 102 isolates had mutations in gyrA gene. While gyrB gene mutations were observed in only two isolates. Mutational analysis showed that the mutations mostly alter protein at codon 94 (D94G) (represents the replacement of aspartic acid with glycine) and 90 (A90V) (substitution of alanine with valine). In MDR and treatment failure cases, the FQs-R was most commonly associated with D94G mutation. Whereas, a high proportion of A90V mutation was observed in MTB isolates which were newly diagnosed.

Conclusion The findings suggest that the genotypic studies for FQs resistance should be carried out at time of initial diagnosis, before starting treatment, to rule out all type of mutations and its potential use in the treatment and to control resistance.

\section{Background}

Pakistan is among the thirty high Tuberculosis (TB) burden countries where complete elimination of TB is, unfortunately, a distant reality. TB is ranked in ninth number with leading cause of mortality worldwide where, about one third of the world's population is infested with latent infection with TB [1]. According to the World health organization (WHO) 2018 annual report, the incidence rate of TB in Pakistan is $267 / 100,000$ population where mortality rate is reported as $27 / 100,000$ (excludes HIV co-infection cases) [2]. There is significant number of population where TB remains undiagnosed and untreated.

The phenotypic resistance to flouroquinolones (FQs) is associated with mutation in $g y r A$ and $g y r B$ gene in quinolone resistance-determining region (QRDR), a type II DNA topoisomerase, which target DNA gyrase. Mutations in the DNA gyr subunit A confer the high level resistance whereas other confers low level resistance to gyr subunit B [3]. FQs have long been using as anti-tuberculosis drugs and their wide spread use has led to the development of resistance in clinical isolates of Mycobacterium tuberculosis (Mtb). During treatment of TB, MDR patients can develop resistance against flouroquinolones. The development of FQs-R in these patients is a risk factor as additional resistance to this drug can aid in transition of these patients from MDR to pre-XDR or even they can become extensively drug resistant with further resistance to at least one injectable second line drug $[4,5]$. 
Geographic differences exist in the frequency of gyrA mutations. The understanding of frequency and geographic distribution of the FQs mutation is important in order to maximize its sensitivity and specificity. Mutations in codon 88-94 of QRDR of gyrA gene appear most commonly particularly in codons $88,90,91$, and 94 . The mutations in the region of codon 500 and 538 of $g y r B$ gene are most often associated with FQs resistance [6].

The emergence of drug resistance and persistence of infection is serious threat to control TB [7]. This high incidence of resistance severely limits treatment options and requires the use of more toxic and costly treatment regimens [8]. The present study is conducted to detect the mutational profile of FQs-R to help in determination of their potential utility and selection of adequate drug regimens.

\section{Methods}

\section{Sample collection}

Mycobacterium tuberculosis isolates were procured from patients diagnosed with pulmonary TB. The samples were collected from PMDT sites (Programmatic management of drug resistant tuberculosis) of seven different districts (Lahore, Faisalabad, Gujranwala, Sahiwal, Sargodha, Sialkot, and Bahawalpur) from period of May 2018 to March 2019 (Fig. S1). A total of 562 suspected MDR-TB cases were included in the study. GeneExpert and MTBDR plus assay was performed for susceptibility against first line anti-TB drugs. History of anti-tuberculosis treatment was obtained from patients which include newly diagnosed cases and previously treated cases (treatment failure and treatment default).

The study was approved by the Research Ethics and Biosafety Committee (No.D/650/MMG) of Department of Microbiology and Molecular Genetics, University of the Punjab, Lahore, Pakistan.

\section{Sample processing}

Initially, the sputum samples were decontaminated using standard NALC-NaOH (N-acetyl-L-cysteine sodium hydroxide) method [9] and smear positive samples were directly processed for DNA extraction. Smear negative samples were primarily cultured on MGIT Bactec $960 \AA$ medium. DNA was extracted using Genolyse version 1.0 kit method (Hain Lifescience, Germany). After DNA extraction, supernatant was collected and transferred into fresh tube and stored at $-20^{\circ} \mathrm{C}$ for further processing.

\section{Molecular detection of FQs resistance}

The phenotypic resistance to second line drugs including flouroquinolones using GenoType MTBDRs/ version 2.0 kit method. The whole procedure of molecular detection with GenoType MTBDRs/ includes three steps i.e. DNA extraction, amplification with biotinylated primers and reverse hybridization. The test was considered valid when all control bands appeared correctly.

\section{PCR and sequencing}


Primers were designed against QRDR region of gyrA gene forward primer 5GATGCAGCGCAGCTACATCGAC-3 and Reverse primer 5-GATGCAGCGCAGCTACATCGAC-3. The cyclic parameters for the amplification reaction were $95^{\circ} \mathrm{C}$ for $5 \mathrm{~min}$ followed by 40 cycles of $95^{\circ} \mathrm{C}$ for $30 \mathrm{sec}$, $61^{\circ} \mathrm{C}$ for $45 \mathrm{sec}, 72^{\circ} \mathrm{C}$ for $50 \mathrm{sec}$ and final elongation for $10 \mathrm{~min}$ at $72^{\circ} \mathrm{C}$. The PCR products were purified using a Qiagen PCR purification kit and eluted in TE buffer. The sequencing of the isolates was performed by | ${ }^{\text {st }}$ Base sequencer, Malaysia.

\section{Results}

GeneExpert assay was performed for the confirmation of MDR-TB. The susceptibility of first line drugs was determined against rifampcin (RIF) and isoniazid (INH). The results have shown that 430 samples were MDR (resistant to both isoniazid and rifampcin), 91 samples were mono-resistant (57 resistant to RIF and 34 resistant to INH), and 41 samples were susceptible to these two drugs.

The analysis of the data showed that of the total 562 cases, 313 were the newly diagnosed, 97 were treatment failure cases (who completed the treatment but still positive for MTB), 59 were treatment default cases (previously taking anti-tuberculosis therapy, at least for one month, but did not complete treatment) and for the 93 samples treatment history was unknown.

\section{Detection of FQs resistance}

The frequency of FQS-R and its mutational profiling was determined in gyr gene. Genotype MTBDRsl assay was performed to determine resistance to second line drugs. Both gyrA and gyrB were examined for FQs resistance. A total of 104/562 (18.5\%) samples were found resistant to FQs.

Among FQs resistant isolates, 8 isolates were rifampcin sensitive, 8 were mono resistant (resistant for rifampcin and sensitive for isoniazid) and 88 were MDR (multi drug resistant). Most of the patients were previously treated and appeared with category 1 failure treatment and some were on category 4 treatment. In total, 102/104 showed the resistance for gyrA gene while gyrB gene mutations were prevalent only in 2/104 cases with mutation in E540V. The resistance was interpreted according to the presence and absence of wild type and mutant probe. When all wild type probes appeared it was interpreted with no detectable mutation. In the absence of any wild type probe, the respective amplicon cannot bind to the corresponding wild type probe resulting in detectable mutation.

\section{Mutational profiling of gyrA gene}

The mutation probes detect some of the most common resistance-mediating mutations. Table 1 shows all types of mutations in the gyrA gene and pattern of corresponding wild and mutant bands. Failure of the gyrA WT3 band and the development of MUT3C was the most common pattern of mutation. Mutational analysis showed that these mutations mostly alter the protein at codon 94 and 90 which represents the replacement of aspartic acid with glycine and alanine with valine, respectively. Aspartic 
acid has also replaced with alanine, asparagine and tyrosine in other types of mutations at codon 94 . Mutation at codon 91 was also found where serine was replaced with proline.

Different hybridization patterns were observed for FQ resistance in gyrA gene, 19 isolates from all possible patterns for further mutational analysis in the hot spot regions of gyrA gene loci using H37RV (AGH06049.1) as a reference strain. In these isolates amino acid changes were determined due to these mutations. We found variation in four isolates with respect to wild type and mutant probe where amino acid change vary from the most known mutation reported by LPA testing. One isolate substituted from asp $\rightarrow$ ala, two isolates had ala $\rightarrow$ val and one had thr $\rightarrow$ ala mutation in addition to ser/ala $\rightarrow X$ (an undetrmined mutation) (Fig. 1).

\section{Discussion}

Fluoroquinolones have long been widely used for several infectious diseases and easily accessible in certain region even without prescription. Such misuse of FQs has highly contributed to their efficacy in the treatment of TB and emergence of FQ-resistance [10].

In the current cross-sectional study, presumptive multi drug resistant isolates of MTB were included. A high proportion of rifampcin (Rif) and isoniazid (INH) resistance was observed. Of the total, $92 \%$ (521/562 i.e. 430 MDR and 91 monoresistant) isolates were resistant to first line drugs (INH and Rif) either both or alone. Among FQs resistant isolates, 8 isolates were rifampcin sensitive, 8 were mono resistant, and 88 were MDR. The isolates having resistance against rifampcin and isoniazid are termed as MDR-TB and if they develop additional resistance against FLs then known as Pre-XDR TB [11].

MTB develop resistance against FQs, mostly, by developing mutations against drug targeted proteins. The detection of gyrase mutations can help in predicting FQs resistance as well as estimating the levels of resistance to various flouroquinolones [12]. GenoType MTBDRs/ assay can detect mutations in the QRDR region of the gyrase gene ( $g y r A$ and $g y r B$. GenoType MTBDRs/ assay was used to determine the frequency of FQs resistance of our isolates. A total of 104/562 (18.5\%) isolates were found resistant to FQs. The high prevalence of FQs resistance was also reported in other provinces of Pakistan $[10,13]$ and neighboring countries India [14,15], China [16,17] and Bangladesh [18,19].

Short treatment regimens are used to reduce emergence of antimicrobial resistance in MTB. According to National guidelines for control of TB in Pakistan 2019 (adopted by WHO), the anti-TB short regimens include third or fourth generation flouroquinolones (levofloxacin and moxifloxacin respectively) for 4 months for drug susceptible cases. They are also given in isoniazid resistant and previously treated cases in initial phase of therapy (2 months). The high proportion of FQ-R indicates the patient ineligibility for shorter regimens.

The resistance to FQs occurs by point mutations in conserved QRDR region of $g y r A$ and $g y r B$ gene. The mutation in QRDR change the structure of drug binding pocket (QBP) of quinolones and results in cross resistance to all FQs. The frequency of gyrA mutations was much high than $g y r B$. It was found in 
hybridization pattern that most of the isolates had mutation in gyrA gene loci with substitution at amino acid 94 and 90 where D94G and A90V were more prevalent. These A90V and D94G mutations are associated with high level of resistance to flouroquinolone antibiotics (Fig. 2). A90V mutation detects resistance for levofloxacin but a higher generation of $F Q$ i.e. moxifloxacin can be used at higher dose. But if there is mutation of D94G both levofloxacin and moxifloxacin are ineffective [20] (GLI guideline Line probe assay). S91P, D94A and D94N/Y were other mutations found in our isolates. With the first two mutations the isolates could be susceptible to moxifloxacin at higher doses but resistant to levofloxacin. However, D94N/Y detect resistance for both levofloxacin and moxifloxacin. These findings correlate with most of the previous studies $[6,21,22]$.

The patient's characteristics and type of mutation is assessed in two ways. The one approach was to determine frequency of particular mutation according to their categorization of resistance to first line drugs and secondly by their categorization according to treatment history. In MDR and RRD cases, the FQs-R was most commonly associated with D94G mutation. This mutation shows high level resistance to all flouroquinolones even to fourth generation moxifloxacin. Monoresistance INH-R showed S91P and D94N/Y. D94N/Y mutation was also observed in RRD. When mutation pattern of newly diagnosed TB isolates with FQs resistance was observed, it showed high proportion of A90V mutation where moxifloxacin still remains the drug of choice at higher doses. In contrast, the mutation pattern of D94G was commonly found in treatment failure and relapse cases.

Since Genotype MTBDRsl assay target only small region of gene with limited number of well-known mutations and sometimes, the interpretations are indistinct for cross-resistance to FQs which occur due to particular gyrA mutations. The sequence analysis was performed for understanding of resistance on the genotypic level [23]. There were some isolates (4/102) which showed co-existence of mutations in hybridization pattern. The combination of mutation was (D94Awith D94H), (S91P with D94G), (D94G with D94N/Y) and (A90V with D94G). Co-existence of mutations was also observed in sequence analysis of gyrA gene where S91T mutation was detected in $95 \%$ isolates. But this type of mutation is not related with flouroquinolone resistance. It could be present even in sensitive isolates as determined by other studies $[24,25]$. The results of all of our mutations are also in line with a study in Pakistan where they detect the mutations in extensively drug resistant strains [26].

Interestingly, we had found some other hot spot mutations in hybridization pattern of co-existence cases where all wild type present with mutant probe. In our two isolates, when MUT3C and MUT2 appeared in the presence of its all wild type probe, substitution of Ala into Val was observed, but when MUT2 probe appeared in the absence of WT3 the mutational change of Asp into Ala occurred. Four isolates selected for our most common pattern i.e. absence WT3 and presence of corresponding mutant probe MUT3C, but, one isolate had shown mutation of Thr into Ala additionally with G/M/R into undetermined amino acid X. For most of the isolates amino acids changed from the pattern of well-known mutations but mostly occurred in the same region i.e. codon 94 and 91 . Overall, these findings suggest that the mutation pattern can differ according to the hybridization pattern of wild type and a mutant probe. 
The study represents the burden of flouroquinolone resistance in MDR-TB patients regardless of FQ antibiotic therapy. However, relevance of genotypic and phenotypic resistance is important to accurately predict FQ-R. Even though we found FQ-R in MTB susceptible isolates but it does not reflect its true prevalence in these patients. The resistance might develop due to the prior use of FQ antibiotic and the results were not included in study.

\section{Conclusions}

In conclusion, the emergence of flouroquinlone resistance in clinical isolates is alarming. We found high proportion of FQs resistance in MDR cases and even in mono-resistant and all drugs sensitive isolates. Previously treated and the failure TB treatment cases being the most important group for developing resistance. Our findings suggest that the implementation of FQs in these patients should be carefully administered and genotypic studies should be carried out, preferably at the time of initial diagnosis, to rule out all type of mutations for effective treatment and particularly to control its resistance.

\section{Abbreviations}

FQ: Flouroquinolone; FQ-R: Flouroquinolone Resistant; LPA: Line Probe Assay; MDR: Multidrug Resistant; MGIT: Mycobacteria Growth Indicator Tube; Mtb: Mycobacterium tuberculosis; MUT: Mutant; NALC-NaOH: $\mathrm{N}$-acetyl-L-Cysteine Sodium Hydroxide; QRDR: Quinolone Resistance Determining Region; TB:

Tuberculosis; WT: Wild Type; XDR: Extensively Drug Resistant.

\section{Declarations}

\section{Ethical approval and consent to participate}

All study participants were informed verbally (approved by Ethics and Biosafety Committee) and consent to participate was collected from the patients directly or from parents. No study participants were included in the present study involving guardians. The study was approved by Departmental Research Ethics and Biosafety Committee.

\section{Consent for publication}

"Not Applicable".

\section{Availability of data and materials}

The datasets generated and/ or analyzed during the current study are part of PhD thesis of the first author and not publicly available. The datasets are available from the corresponding author on reasonable request.

\section{Competing interests}


The authors declare that they have no competing interests.

\section{Funding}

No funding was obtained for the current study.

\section{Authors' contributions}

SK performed experiments, analyzed the data and wrote the manuscript.conceived the research idea. ZT provided sources. NM helped in experiments and data analysis. Both MS had responsibility for data collection. AR conceived the research idea and helped in manuscript preparation. All authors contributed substantially to the interpretation of the results. All authors approved the final manuscript.

\section{Acknowledgements}

"Not Applicable".

\section{References}

[1] Muñoz L, Stagg HR, Abubakar I Diagnosis and management of latent tuberculosis infection. Cold Spring Harb Perspect Med. 2015;5(11): a017830.

[2] WHO Global tuberculosis report 2018. Available from URL https://www.who.int/tb/publications/global_report/gtbr2018_annex3.pdf?ua=1

[3] Zhang Y, Yew WW. Mechanisms of drug resistance in Mycobacterium tuberculosis [State of the art series. Drug-resistant tuberculosis. Edited by CY. Chiang. Number 1 in the series]. Int J Tuberc Lung Dis. 2009;13(11):1320-1330.

[4] Castro RA, Ross A, Kamwela L, Reinhard M, Loiseau C, Feldmann J, Borrell S, Trauner A, Gagneux S. The evolution of fluoroquinolone-resistance in Mycobacterium tuberculosis is modulated by the genetic background. bioRXiv. 2019;2019: 659045.

[5] Sharma R, Sharma SK, Singh BK, Mittal A, Kumar P. High degree of fluoroquinolone resistance among pulmonary tuberculosis patients in New Delhi, India. Indian J Med Res. 2019;149(1): 62-66.

[6] Avalos E, Catanzaro D, Catanzaro A, Ganiats T, Brodine S, Alcaraz J, Rodwell T. Frequency and geographic distribution of $g y r A$ and $g y r B$ mutations associated with fluoroquinolone resistance in clinical Mycobacterium tuberculosis isolates: a systematic review. PLoS One 2015;10(3): e0120470.

[7] Kaur D, Kutum R, Dash D, Brahmachari SK. Data Intensive genome level analysis for identifying novel, non-toxic drug targets for multidrug resistant Mycobacterium tuberculosis. Sci Rep. 2017;7: 46595.

[8] Shah NS, Auld SC, Brust JC, Mathema B, Ismail N, Moodley P, Mlisana K, Allana S, Campbell A, Mthiyane T, Morris N, Mpangase P, van der Meulen H, Omar SV, Brown TS, Narechania A, Shaskina E, 
Kapwata T, Kreiswirth B, Gandhi NR. Transmission of extensively drug-resistant tuberculosis in South Africa. N Engl J Med. 2017;376(3): 243-253.

[9] Stinson KW, Eisenach K, Kayes S, Matsumoto M, Siddiqi S, Nakashima S. et al. Mycobacteriology Laboratory Manual, global laboratory initiative advancing TB diagnosis. $1^{\text {st }} e d$. April 2014.

[10] Jabeen K, Shakoor S, Malik F, Hasan R (2015) Fluoroquinolone resistance in Mycobacterium tuberculosis isolates from Pakistan 2010-2014: Implications for disease control. Int J Mycobacteriol. 2015;4:47-48.

[11] Daniel O, Osman E, Oladimeji O, Dairo OG. Preextensive drug resistant tuberculosis (Pre-XDR-TB) among MDR-TB patents in Nigeria. Global Adv Res J Microbiol. 2013; 2: 22-25.

[12] Dookie N, Rambaran S, Padayatchi N, Mahomed S, Naidoo K. Evolution of drug resistance in Mycobacterium tuberculosis: a review on the molecular determinants of resistance and implications for personalized care. J Antimicrob Chemother. 2018;73(5): 1138-1151.

[13] Akhtar AM, Arif MA, Kanwal S, Majeed S. Prevalence and drug resistance pattern of MDR TB in retreatment cases of Punjab, Pakistan. J Pak Med Assoc. 2016;66(8): 989-3.

[14] Desai U, Joshi JM. Extrapulmonary drug-resistant tuberculosis at a drug-resistant tuberculosis center, Mumbai: Our experience-Hope in the midst of despair! Lung India 2019;36(1): 3-7.

[15] Sharma AK, Gupta N, Kala DK, Patni T, Dixit R, Verma S, Chandran A. A study on pattern of resistance to second line anti tubercular drugs among multi drug resistant tuberculosis patients. Indian $\mathrm{J}$ Tuberc. 2018;65(3): 233-236.

[16] Gao Y, Zhang Z, Deng J, Mansjö M, Ning Z, Li Y, Xu B. Multi-center evaluation of GenoType MTBDRsl line probe assay for rapid detection of preXDR and XDR Mycobacterium tuberculosis in China. J Infect. 2018;77(4): 328-334.

[17] Wang Z, Xie T, Mu C, Sun R, Wang C, Zhao H, Ju H. Performance of sequencing in predicting ofloxacin resistance in Mycobacterium tuberculosis from positive Bactec MGIT 960 cultures. Ann Clin Lab Sci. 2018;48(1): 69-74.

[18] Rigouts L, Coeck N, Gumusboga M, de Rijk WB, Aung KJM, Hossain MA, Van Deun A. Specific gyrA gene mutations predict poor treatment outcome in MDR-TB. J Antimicrob Chemother. 2015;71(2): 314323.

[19] Kamal SMM, Hossain A, Sultana S, Begum V, Haque N, Ahmed J, Chowdhury RA. Anti-tuberculosis drug resistance in Bangladesh: reflections from the first nationwide survey. Int. J Tuberc Lung Dis. 2015;19(2): 151-156. 
[20] Mogashoa T, Melamu P, Derendinger B, Ley SD, Streicher EM, Iketleng T., Mupfumi L, Mokomane M, Kgwaadira B, Rankgoane-Pono G, Tsholofelo TT, Kasvosve I, Moyo S, Warren RM, Gaseitsiwe S. Detection of second line drug resistance among drug resistant Mycobacterium tuberculosis isolates in Botswana. Pathogens 2019;8(4): E208.

[21] Von Groll A, Martin A, Jureen P, Hoffner S, Vandamme P, Portaels F, Palomino JC, da Silva PA. Fluoroquinolone resistance in Mycobacterium tuberculosis and mutations in gyrA and gyrB. Antimicrob Agents Chemother. 2009;53(10): 4498-4500.

[22] Singhal R, Reynolds PR, Marola JL, Epperson LE, Arora J, Sarin R, Myneedu VP, Strong M, Salfinger $M$. Sequence analysis of fluoroquinolone resistance-associated genes gyrA and gyrB in clinical Mycobacterium tuberculosis isolates from patients suspected of having multidrug-resistant tuberculosis in New Delhi, India. J Clin Microbiol. 2016;54(9): 2298-2305.

[23] Schön T, Miotto P, Köser CU, Viveiros M, Böttger E, Cambau E. Mycobacterium tuberculosis drugresistance testing: challenges, recent developments and perspectives. Clin Microbiol Infect. 2017;23(3):154-160.

[24] Giannoni F, Iona E, Sementilli F, Brunori L, Pardini M., Migliori B, Orefici G, Fattorini L. Evaluation of a new line probe assay for rapid identification of gyrA mutations in Mycobacterium tuberculosis. Antimicrob Agents Chemother. 2005;49(7):2928-2933.

[25] Juarez-Eusebio DM, Munro-Rojas D, Muñiz-Salazar R, Laniado-Laborín R, Martinez-Guarneros JA, Flores-López CA, Zenteno-Cuevas R. Molecular characterization of multidrug-resistant Mycobacterium tuberculosis isolates from high prevalence tuberculosis states in Mexico. Infect Genet Evol. 2017;55: 384391.

[26] Ali A, Hasan R, Jabeen K, Jabee N, Qadeer E, Hasan Z. Characterization of mutations conferring extensive drug resistance to Mycobacterium tuberculosis isolates in Pakistan. Antimicrob Agents chemother. 2011;55(12):5654-5659.

\section{Table}

Table 1: The frequency and the mutations conferring resistance to FQs. 


\begin{tabular}{llllc}
\hline $\begin{array}{l}\text { gyrA Mutation } \\
\text { probe }\end{array}$ & $\begin{array}{l}\text { Missing wild type } \\
\text { probe }\end{array}$ & $\begin{array}{l}\text { Phenotypic } \\
\text { Susceptibility }\end{array}$ & Mutation & $\begin{array}{l}\text { Frequency } \\
(\mathbf{n = 1 0 2})\end{array}$ \\
\hline gyr A MUT1 & gyr A WT2 & Resistant & A90V & 25 \\
\hline gyr A MUT2 & gyr A WT2 & Resistant & S91P & 7 \\
& gyr A WT3 & & & 7 \\
\hline gyr A MUT3A & gyr A WT3 & Resistant & D94A & 9 \\
\hline gyr A MUT3B & gyr A WT3 & Resistant & D94N/Y & 45 \\
\hline gyr A MUT3C & gyr A WT3 & Resistant & D94G & 9 \\
\hline None & $\begin{array}{l}\text { any one wild type } \\
\text { probe }\end{array}$ & Resistant & A90V D94A \\
& & D94G & \\
\hline
\end{tabular}

\section{Figures}

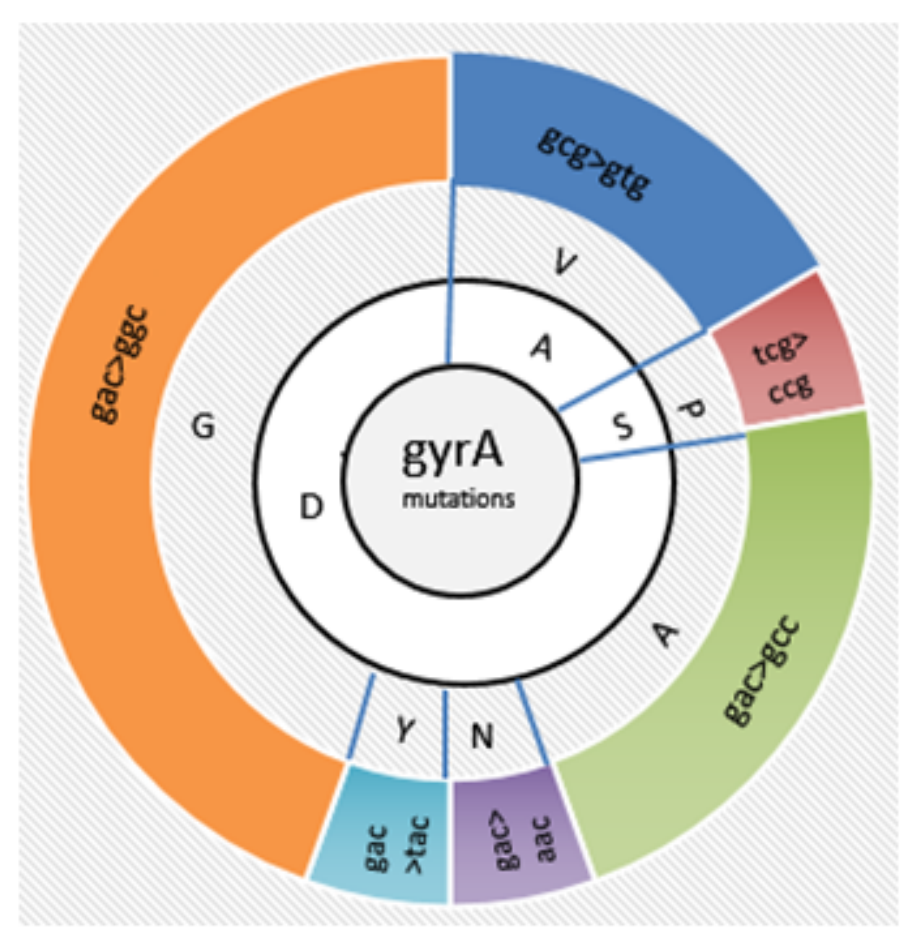

\section{Figure 1}

Frequency amino acid and nucleotide change in FQs isolates. In general, amino acid change had found in three amino acids alanine ( $A$ into $V$ ), serine ( $S$ into $P$ ) and aspartic acid ( $D$ either into $A, N, Y$ or $G$ ). The nucleoide change corresponding to these amino acids change is also represented. 


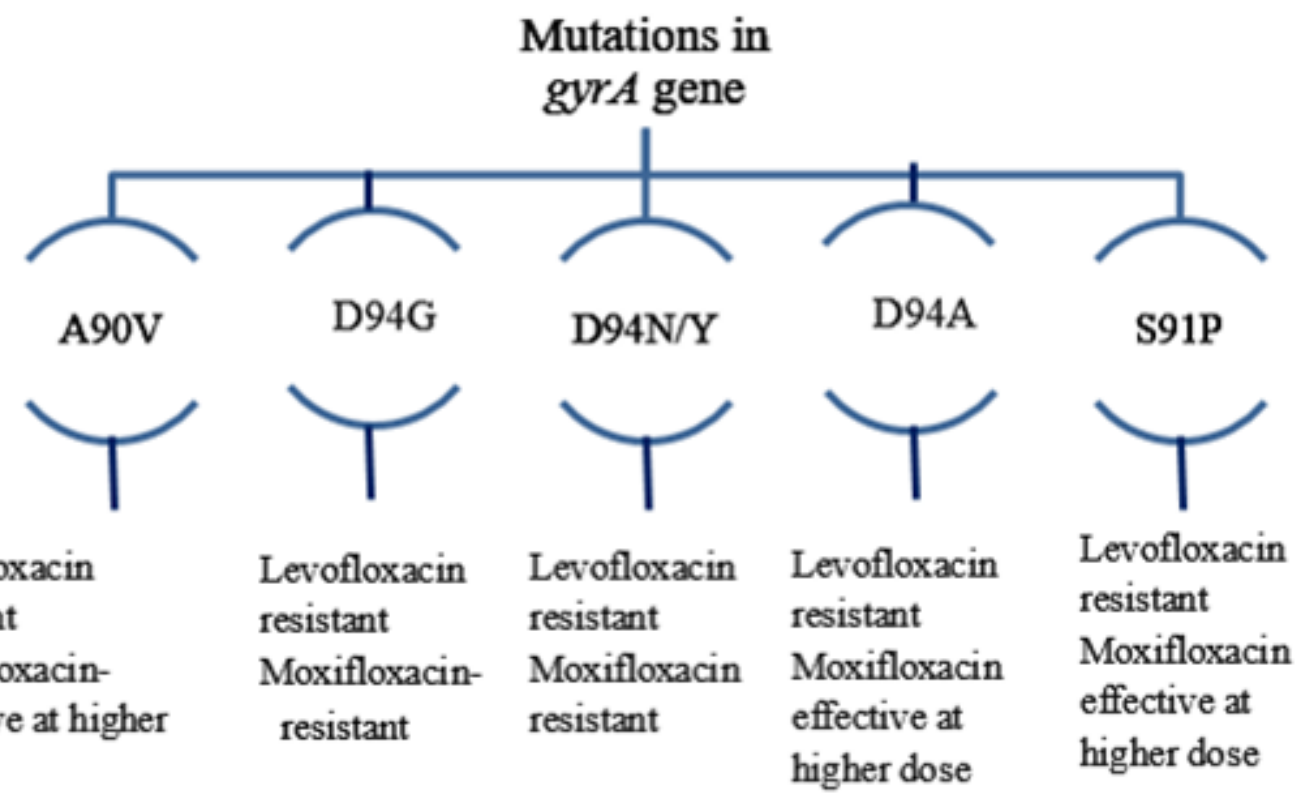

\section{Figure 2}

The association of gyrA gene mutation with levofloxacin and moxifloxacin resistance.

\section{Supplementary Files}

This is a list of supplementary files associated with this preprint. Click to download.

- SupplementarydataBMCPulMed.docx 